\title{
New universality class for the fragmentation of plastic materials
}

\author{
G. Timár, ${ }^{1,2}$ J. Blömer, ${ }^{3}$ F. Kun, ${ }^{1}$ and H. J. Herrmann ${ }^{2,4}$ \\ ${ }^{1}$ Department of Theoretical Physics, University of Debrecen, P. O. Box:5, H-4010 Debrecen, Hungary \\ ${ }^{2}$ Computational Physics IfB, HIF, ETH, Hönggerberg, 8093 Zürich, Switzerland \\ ${ }^{3}$ Spezialwerkstoffe, Fraunhofer UMSICHT Osterfelder Str. 3, 46047 Oberhausen, Germany \\ ${ }^{4}$ Departamento de Fisica, Universidade Federal do Ceara, 60451-970 Fortaleza, Ceara, Brazil
}

(Dated: August 6, 2018)

\begin{abstract}
We present an experimental and theoretical study of the fragmentation of polymeric materials by impacting polypropylene particles of spherical shape against a hard wall. Experiments reveal a power law mass distribution of fragments with an exponent close to 1.2 , which is significantly different from the known exponents of three-dimensional bulk materials. A 3D discrete element model is introduced which reproduces both the large permanent deformation of the polymer during impact, and the novel value of the mass distribution exponent. We demonstrate that the dominance of shear in the crack formation and the plastic response of the material are the key features which give rise to the emergence of the novel universality class of fragmentation phenomena.
\end{abstract}

PACS numbers: 62.20.Mk; 46.50.+a; 64.60.-i

Fragmentation phenomena are ubiquitous in nature and play a crucial role in numerous industrial processes related to mining and ore processing [1]. A large variety of measurements starting from the breakup of heavy nuclei through the usage of explosives in mining or fragmenting asteroids revealed the existence of a striking universality in fragmentation phenomena [1 10]: fragment mass distributions exhibit a power law decay, independent on the type of energy input (impact, explosion, ...), the relevant length scales or the dominating microscopic interactions involved. Detailed laboratory experiments on the breakup of disordered solids have revealed that mainly the effective dimensionality of the system determines the value of the exponent, according to which universality classes of fragmentation phenomena can be distinguished. Several possible mechanisms have been put forward to understand the emergence of the universal power law behavior. For rapid break-up of heterogeneous bulk solids with a high degree of brittleness, the self-similar branching-merging scenario of propagating unstable cracks governed by tensile stresses can explain the main features of the fragment mass distribution 5, 11 13], while for shell systems an additional sequential binary breakup mechanism has to be taken into account [7, 8]. It is an important question of broad scientific and technological interest how plasticity, and the emergence of complicated stress states like shear affect the fragmentation process. The fundamental questions of how robust the universality classes are with respect to mechanical properties and whether there exist further universality classes of fragmentation of solids, still remain open.

In the present Letter we investigate the fragmentation process of plastic materials by impacting spherical particles made of polypropylene (PP) against a hard wall. Our experiments show that the mass distribution of plastic fragments exhibits a power law behavior with an exponent close to 1.2 , which is substantially different from the
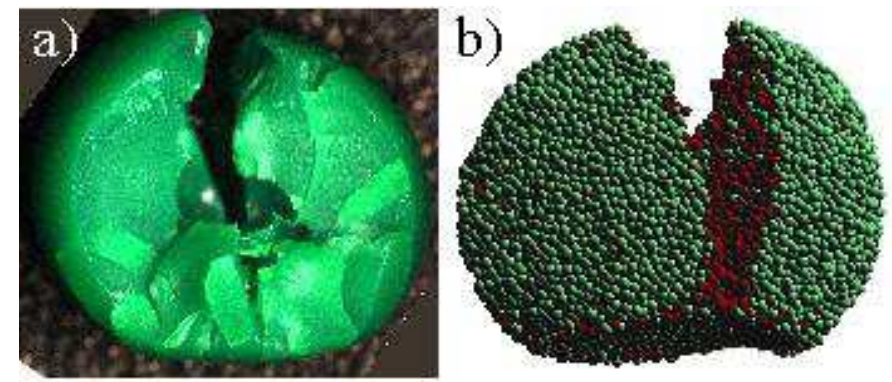

FIG. 1: (Color online) Final states of impact at low impact velocities in the experiment $(a)$ and in the simulation $(b)$. In the contact area with the hard wall large permanent deformations occur due to compression, while above it vertical cracks are formed due to tensile stresses. The simulations are in very good agreement with the measurements.

one of bulk brittle materials in three-dimensions. In order to understand the physical origin of the low exponent, a three-dimensional discrete element model is developed where the sample is discretized in terms of spherical particles connected by elastic beams. To capture the fracture mechanisms of plastic materials in the model, broken particle contacts are able to reconnect when compressed against each other leading eventually to the healing of cracks and to a plastic energy dissipation. By adding two novel ingredients into a DEM model, namely healing of broken bonds and breaking under compression, we are able to simulate the plastic behavior of $\mathrm{PP}$ and reproduce in fact the observed new power law distribution.

In the experiments we used isotactic polypropylene, which is a thermoplastic polymer with an intermediate level of crystallinity in its molecular structure. The most important parameters of PP are: Young modulus 1300 $\mathrm{MPa}$ (room temperature), glass transition temperature $-10{ }^{\circ} \mathrm{C}$, melting point $160{ }^{\circ} \mathrm{C}$, and density $0.9 \mathrm{~g} / \mathrm{cm}^{3}$. Besides tacticity, the mechanical response and fracture characteristics of PP are also strongly affected both by 
the temperature and by the rate of loading: increasing strain rate gives rise to a more brittle response while the raising temperature enhances ductility [14]. To achieve fragmentation a single particle comminution device was used which accelerates particles one-by-one by centrifugal force in a rotor up to the desired velocity. The rotor ensures that the particles hit the hard wall at a rectangular angle in an evacuated environment eliminating the disturbing effect of inclined impact and of turbulent air flow. Spherical PP particles of diameter $d=4 \mathrm{~mm}$ were fragmented at different impact velocities $v_{0}$ in the range $30 \mathrm{~m} / \mathrm{s}-180 \mathrm{~m} / \mathrm{s}$. Figure 1(a) shows that at low enough velocities, the collision does not result in a breakup, instead the particles undergo a large plastic deformation at the impact site. Above the completely flattened contact zone of permanent deformation meridional cracks form due to tensile stresses, however, the body does not fall apart, the plastic zone retains its integrity.

Fragmentation occurs when the impact velocity $v_{0}$ exceeds a material dependent critical value $v_{c}$, which is about $60 \mathrm{~m} / \mathrm{s}$ for our PP particles. At each impact velocity 400 particles were fragmented accumulating the fragments in the grinding chamber of the machine. In the data analysis, $99-99.5 \%$ of the total mass of the samples was recovered. In order to evaluate the mass distribution of the fragments, we scanned the pieces with an open scanner obtaining digital images where fragments appear as white spots on the black background [7, 8]. This way the identification of fragments is reduced to cluster searching of white pixels. The very fine powder of extension smaller then the pixel size of the scanner was left out of the data analysis. The two-dimensional projected area $w$ of fragments is determined as the number of pixels of the clusters, from which the mass $m$ of fragments can be estimated as $m \sim w^{3 / 2}$ since the threedimensional fragment shape is close to isotropic. We did check the shape isotropy by calculating the square root of the ratio of the larger $I_{1}$ and smaller $I_{2}$ eigenvalues of the tensor of inertia of the $2 \mathrm{D}$ projections. The inset of Fig. 2 shows that the value of $\sqrt{I_{1} / I_{2}}$ is close to 1.9 for almost all fragment masses indicating a high level of isotropy. The mass distribution $F(m)$ of fragments obtained at three different impact velocities is presented in Fig. 2. It can be observed in the figure that at the highest impact velocity $v_{0}=75 \mathrm{~m} / \mathrm{s}$, where the state of complete breakup is reached, a power law functional form emerges

$$
F(m) \sim m^{-\tau_{p l}}
$$

over more than 3 orders of magnitude in the regime of small fragments. At lower impact velocities the power law regime of the distribution is followed by a hump for the largest fragments which gradually disappears and the cutoff becomes exponential as $v_{0}$ increases. The most astonishing feature of the experimental results is that the value of the exponent $\tau_{p l}=1.2 \pm 0.06$ of the power law regime is significantly lower than the values $\tau_{b r} \approx$

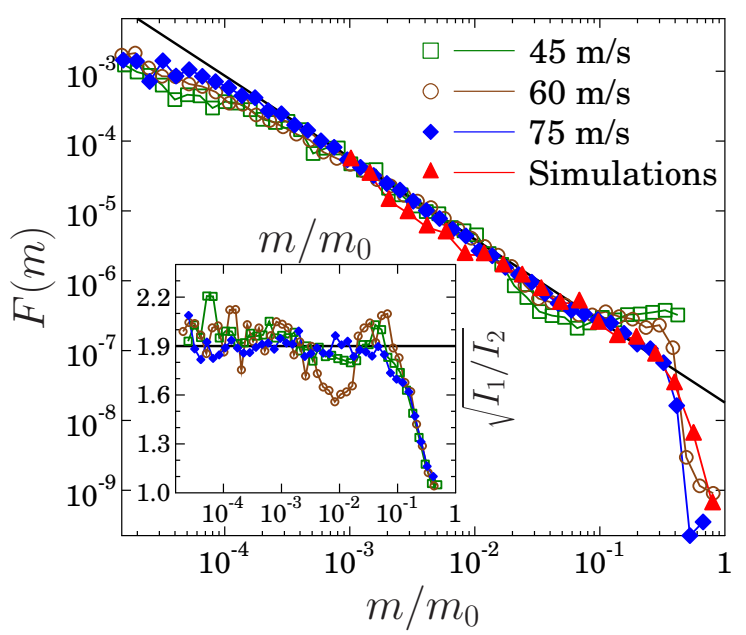

FIG. 2: (Color online) Mass distribution of fragments obtained from experiments at three different impact velocities. $m_{0}$ denotes the average mass of PP spheres. At the highest impact velocity $v_{0}=75 \mathrm{~m} / \mathrm{s}, F(m)$ shows a power law behavior over 3 orders of magnitude followed by an exponential cutoff for the very large pieces. Simulation results obtained with the parameters $\Theta_{t h}=1$ and $t_{h}=0$ (bendingÉhealing) are in a very good agreement with the experimental findings. Inset: the shape parameter $\sqrt{I_{1} / I_{2}}$ of fragments as a function of mass for the experiments of the main figure.

$1.8-2.1$ typically found in the fragmentation of threedimensional bulk objects consisting of disordered brittle materials 36 , 6,12$]$. The anomalously low value of $\tau_{p l}$ is the consequence of the breakup mechanism of plastic materials which has not been considered by the usual theoretical approaches [6, 12].

In order to reveal the underlying physical mechanisms of the fragmentation of plastic materials, we used a Discrete Element Model (DEM) to simulate the fragmentation of polymeric particles of spherical shape when they impact a hard wall. The spherical sample is represented as a random packing of spheres with a narrow size distribution. The particles are connected by beams along the edges of a Delaunay triangulation of the initial particle positions such that in the initial state neighboring elements are in contact. In 3D the total deformation of a beam is calculated by the superposition of elongation, torsion, as well as bending and shearing in two different planes [15]. To capture one crucial aspect of the deformation behavior and the fracture of plastic materials, our DEM model has two novel components, i.e. the form of the breaking criterion of the beam elements and the reactivation of broken contacts under pressure. The beams break when they get overstressed according to a physical breaking rule [16]

$$
\frac{\varepsilon|\varepsilon|}{\varepsilon_{t h}^{2}}+\frac{\max \left(\left|\Theta_{i}\right|,\left|\Theta_{j}\right|\right)}{\Theta_{t h}} \geq 1,
$$

where $\varepsilon$ denotes the longitudinal strain, $\Theta_{i}$ and $\Theta_{j}$ are the generalized bending angles at the two beam ends, and $\varepsilon_{t h}$ 

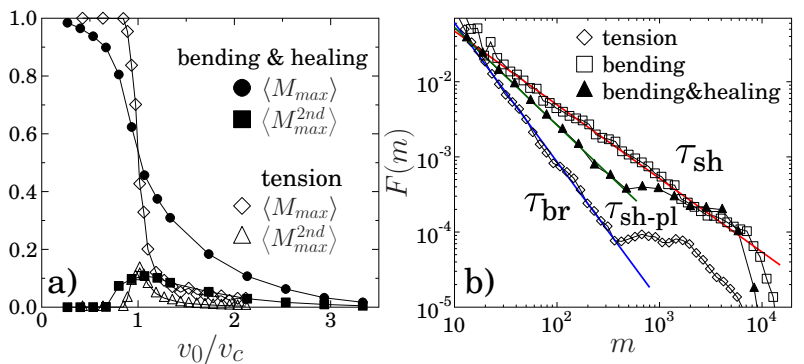

FIG. 3: (Color online) (a) The average mass of the largest $\left\langle M_{\max }\right\rangle$ and second largest $\left\langle M_{\max }^{2 n d}\right\rangle$ fragments as a function of the impact velocity $v_{0}$ normalized by the critical velocity $v_{c}$ of fragmentation for two parameter sets. (b) Fragment mass distributions obtained for the limiting cases of tension, bending, and bending\&healing dominated breakups giving the corresponding exponents $\tau_{b r}, \tau_{s h}$, and $\tau_{s h-p l}$, respectively.

and $\Theta_{t h}$ are breaking thresholds which have fixed values for all the beams. The two terms of Eq. (2) characterize the contributions of the stretching and bending failure modes of a beam. Simulations showed that the local shear of the particle contacts provides the main contribution to the bending angles $\Theta_{i}, \Theta_{j}$, so that bending dominated beam breaking in Eq. (2) characterizes crack formation due to shear. Varying the values of the breaking thresholds $\varepsilon_{t h}$ and $\Theta_{t h}$, the relative importance of stretching and bending can be controlled: increasing the value of a breaking parameter, the effect of the corresponding failure mode diminishes. An important feature of the breaking criterion is that in Eq. (2) the deformation $\varepsilon$ is not restricted to positive values contrary to former simulation studies on brittle fracture and fragmentation [7, 8, 11]. Since the first term of Eq. (2) becomes negative when the beam is compressed, failure is dominated by the bending/shear mode in such a way that increasing compression increases the shear resistance of the beam. In order to represent the plastic behavior of the material, we assume that the beams have a linear elastic behavior up to fracture, but, whenever two particles are pressed against one another for a time longer than $t_{h}$, a new, undeformed beam is inserted between them. This way during the impact process, the particle contacts may undergo a sequence of breaking-healing events which leads to plastic energy dissipation and to the appearance of permanent deformation. Varying the healing time $t_{h}$ the mechanical response of the model material can be controlled: $t_{h}=0$ corresponds to the case of perfect shear plasticity, while $t_{h} \rightarrow \infty$ implies no healing at all, i.e. brittle behavior.

In order to investigate the effect of local failure modes of beams on the fragmentation process, we carried out computer simulations by setting the stretching threshold to a fixed value $\varepsilon_{t h}=0.02$ and varying the bending threshold within a broad range $1.0 \leq \Theta_{t h} \leq 200$. In this way $\Theta_{t h}=200$ and $\Theta_{t h}=1$ imply total tension and bending dominance, respectively, while intermediate $\Theta_{t h}$

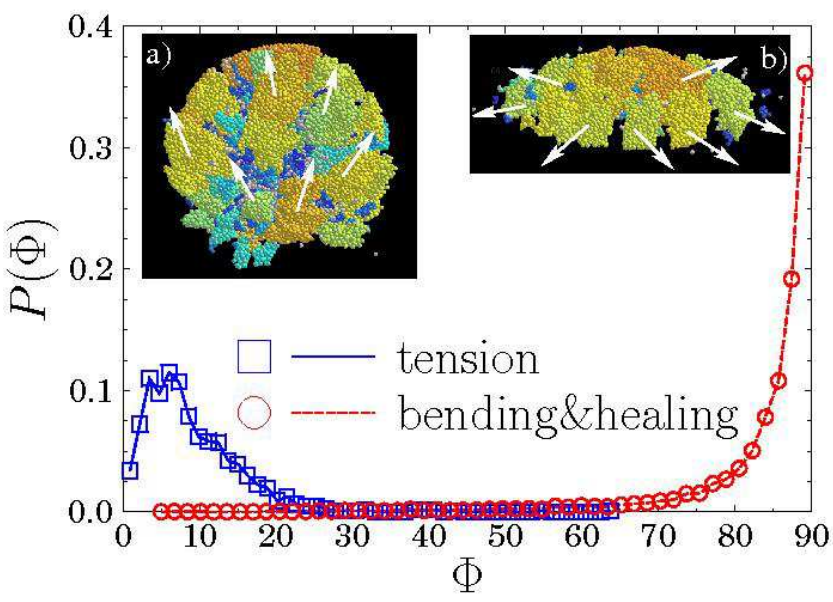

FIG. 4: (Color online) Probability distribution of the angle $\Phi$ between the velocity vector of fragments and the direction of impact for tension $\Theta_{t h}=200$ and shear $\Theta_{t h}=1.0$ dominated breakup. Insets: final states of fragmentation for tension $(a)$ and shear $(b)$ dominance. The arrows indicate the direction of the velocity of a few fragments.

values interpolate between the two limits. Simulations were stopped when the system reached a relaxed state, i.e. no beam breaking occurred during 1000 consecutive time steps. Figure 1 $(b)$ presents the final state of an impact simulation obtained with a sample of $N=24000$ particles at low impact velocity. It can be observed that the model is able to reproduce both the deformation and the crack structure of PP, with parameter values where the beam breaking is dominated by bending $\Theta_{t h}=1.0$, furthermore, compressed contacts easily heal $t_{h}=0$. The large permanent deformation of the sphere in Fig. 1 $(b)$ arises due to breaking-healing sequences of particle contacts in the compressed impact zone. Above this zone tensile stresses arise resulting in opening cracks along the impact direction in agreement with the experiments. Figure $3(a)$ presents the average mass of the largest $\left\langle M_{\max }\right\rangle$ and second largest $\left\langle M_{\max }^{2 n d}\right\rangle$ fragments as a function of the impact velocity $v_{0}$ for the tension $\Theta_{t h}=200$ dominated brittle breakup $t_{h}=\infty$ (tension), and for the bending dominated fragmentation $\Theta_{t h}=1.0$ with perfect shear plasticity $t_{h}=0$ (bending\&healing). For low impact velocities the sample gets only damaged, hence, the largest fragment comprises nearly the entire mass $M_{0}$ of the body $\left\langle M_{\max }\right\rangle \sim M_{0}$, while the second largest one is orders of magnitude smaller $\left\langle M_{\max }^{2 n d}\right\rangle \ll\left\langle M_{\max }\right\rangle$. Fragmentation occurs when the largest and second largest pieces become comparable, i.e. at the critical velocity $v_{c}$ where the $\left\langle M_{\max }^{2 n d}\right\rangle$ curve has a maximum coinciding with the inflexion point of $\left\langle M_{\max }\right\rangle$. Figure 3( $(a)$ shows that for the breakup of brittle materials dominated by tensile stresses, the damage-fragmentation transition is sharp in agreement with experiments 9, 10, however, when shear breaking dominates a broad critical regime emerges. The reason is that at $v_{c}$ the largest fragment does not break 
up into large pieces as for the tension case, but instead gradually erodes with a cleavage mechanism giving rise to a slowly decaying residue.

Representative examples for the mass distribution of fragments are presented in Fig. 3(b) for three parameter sets. For the mass distribution of the tension dominated breakup of heterogeneous brittle materials obtained at the parameter values $\Theta_{t h}=200, t_{h}=\infty$ (tension) a power law behavior is evidenced with the usual exponent $\tau_{b r}=1.9 \pm 0.1[15]$. Simulations showed that decreasing the healing time $t_{h} \rightarrow 0$ in the tension limit practically does not affect the fragmentation process because fragments are only generated by opening cracks which do not let healing play any role. It is important to emphasize that the breakup process substantially changes when shear dominates the crack formation $\Theta_{t h}=1$ (bending). Even in the case of perfectly brittle beam breaking $t_{h}=\infty$ the low shear resistance leads to a significantly lower exponent than in the tensile limit $\tau_{s h}=1.0 \pm 0.05$ (see Fig. $3(b)$ ). Increasing the strength of plasticity $t_{h} \rightarrow 0$ when shear dominates the cracking $\Theta_{t h}=1$, the exponent of the fragment mass distribution slightly increases: due to the healing of cracks fragments can merge which decreases the relative frequency of large pieces leading to a faster decay of the distribution. The highest value of the exponent $\tau_{s h-p l}=1.25 \pm 0.06$ is obtained in the plastic limit $t_{h}=0$ together with $\Theta_{t h}=1.0$ (bending 6 healing). It is important to emphasize that varying solely the velocity of impact in Fig. 2 for the mass distribution of fragments an excellent agreement is obtained between the shear-plastic simulations and the experiments. The results show that the shear dominated cracking together with the healing mechanism of compressed crack surfaces are responsible for the unique fragmentation of plastic materials. During the impact process of the experiments a considerable fraction of the kinetic energy of the particle gets transformed into heat which enhances the effect of healing and plasticity in consistence with our theoretical results. Our extensive simulations indicate that the exponent $\tau_{s h-p l}$ is universal, i.e. it does not depend on the impact velocity or on materials' microstructure characterizing a novel universality class of fragmentation phenomena.

Due to low shear resistance, we find that the fragmentation of plastic shows similarities to the breakup of liquid droplets colliding with a wall. The spatial distribution of fragments and the crack structure in the final state of brittle and plastic fragmentation are compared in the insets of Fig. (4) Although meridional and segmentation cracks are clearly observed in the brittle case in agreement with experiments, a lateral spreading of fragments is obtained for plastic with shear dominated breaking. For the quantitative characterization of the spatial spread of fragments, the distribution of the angle $\Phi$ of the velocity vector of fragments with the impact direction (verti-

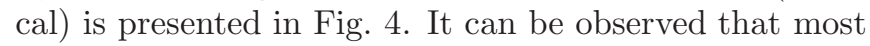

of the fragments bounce back from the hard wall when tension dominates, however, in the shear-plastic case the fragments escape laterally producing a "splash" of the entire body similar to liquid droplets [17, 18].

In conclusion, our experimental and theoretical study revealed that the breakup of plastic materials falls into a novel universality class of fragmentation phenomena characterized by the new mass distribution exponent. Based on discrete element simulations we showed that the plastic behavior of the material together with the dominance of shear in crack formation are responsible for the substantial difference from brittle fragmentation. The low shear resistance of the material gives rise to a splashing similar to the breakup of droplets of highly viscous liquids. Beyond the industrial importance of the fragmentation of polymeric materials, our results might also be applied to obtain a deeper understanding of the fragmentation of highly viscous magma during pyroclastic activity at volcanic eruption [19, 20]. For theoretical investigations our results demonstrate that the breakup of solids cannot be understood as a generic stochastic process since the precise mechanism of crack initiation and growth, i.e. the dominance of tensile or shear stresses govern the exponent of fragmentation. Still remains the challenge to construct a theoretical approach which explains the emergence of universality based on crack dynamics.

The project was partially supported by the German Federal Ministry of Economics and Technology (BMWi) via AiF-Grant-No. 14516 N. F. K. acknowledges the financial support of the Janos Bolyai Grant of HAS.

[1] J. A. Aström, Adv. in Phys. 55, 247 (2006).

[2] D. L. Turcotte, J. of Geophys. Res. 91, 1921 (1986).

[3] L. Oddershede, P. Dimon, and J. Bohr, Phys. Rev. Lett. 71, 3107 (1993).

[4] A. Meibom and I. Balslev, Phys. Rev. Lett. 76, 2492 (1996).

[5] J. A. Aström, R. P. Linna, J. Timonen, P. F. Möller, and L. Oddershede, Phys. Rev. E 70, 026104 (2004).

[6] J. Astrom, A. Holian, and J. Timonen, Phys. Rev. Lett. 84, 3061 (2000).

[7] F. Wittel, F. Kun, H. J. Herrmann, and B. H. Kröplin, Phys. Rev. Lett. 93, 035504 (2004).

[8] F. Kun, F. K. Wittel, H. J. Herrmann, B.-H. Kröplin, and K. J. Maloy, Phys. Rev. Lett. 96, 025504 (2006).

[9] H. Katsuragi, S. Ihara, and H. Honjo, Phys. Rev. Lett. 95, 095503 (2005).

[10] T. Kadono, Phys. Rev. Lett. 78, 1444 (1997).

[11] J. Aström and J. Timonen, Phys. Rev. E 78, 3677 (1997).

[12] J. A. Aström, F. Ouchterlony, R. P. Linna, and J. Timonen, Phys. Rev. Lett. 92, 245506 (2004).

[13] P. Kekalainen, J. A. Astrom, and J. Timonen, Phys. Rev. E 76, 026112 (2007).

[14] H. Saechtling, J. Haim, and D. Hyatt, Saechtling International Plastics Handbook: For the Technologist, En- 
gineer and User (Hanser Gardner Publications, 1995), chap. 3.3.1.

[15] H. A. Carmona, F. K. Wittel, F. Kun, and H. J. Herrmann, Phys. Rev. E 77, 051302 (2008).

[16] H. J. Herrmann, A. Hansen, and S. Roux, Phys. Rev. B 39, 637 (1989).

[17] E. Villermaux, Annu. Rev. Fluid Mech. 39, 419 (2007).
[18] C. F. Moukarzel, S. F. Fernandez-Sabido, and J. C. RuizSuarez, Phys. Rev. E 75, 061127 (2007).

[19] I. Sugioka and M. Bursik, Nature 373, 689 (1995).

[20] M. Alidibirov and D. B. Dingwell, Nature 380, 146 (1996). 